\title{
CHEVALLEY'S AMBIGUOUS CLASS NUMBER FORMULA FOR AN ARBITRARY TORUS
}

\author{
Cristian D. GonzÁlez-Avilés
}

\begin{abstract}
In this paper we obtain Chevalley's ambiguous class number formula for an arbitrary torus $T$ over a global field. The classical formula of C.Chevalley may be recovered by setting $T=\mathbb{G}_{m}$ in our formula. A key ingredient of the proof is the work of X.Xarles on groups of components of Néron-Raynaud models of tori.
\end{abstract}

\section{Introduction}

Let $F$ be a global field and let $S$ be a nonempty finite set of primes of $F$ containing the archimedean primes in the number field case. Further, let $C_{F, S}$ denote the $S$-ideal class group of $F$ and let $K / F$ be a finite Galois extension with Galois group $G$. We will write $C_{K, S_{K}}$ for the $S_{K}$-ideal class group of $K$, where $S_{K}$ is the set of primes of $K$ lying above the primes in $S$. Now let $\mathcal{O}_{K, S_{K}}$ denote the ring of $S_{K}$-integers of $K$ and, for each prime $v$ of $F$, let $e_{v}$ denote the ramification index of $v$ in $K / F$. The order of a finite set $M$ will be denoted by $[M]$.

In his 1933 thesis [6, C.Chevalley obtained the following ambiguous class number formula, which has become well-known.

Theorem 1.1. We have

$$
\frac{\left[C_{K, S_{K}}^{G}\right]}{\left[C_{F, S}\right]}=\frac{\left[H^{1}\left(G, K^{*} / \mathcal{O}_{K, S_{K}}^{*}\right)\right]}{\left[H^{1}\left(G, \mathcal{O}_{K, S_{K}}^{*}\right)\right]} \prod_{v \notin S} e_{v} .
$$

In this paper we extend the above formula to an arbitrary torus $T$ over $F$ (the preceding formula may then be recovered from our Main Theorem below by setting $T=\mathbb{G}_{m, F}$ there).

Let $\widetilde{U}=\operatorname{Spec} \mathcal{O}_{K, S_{K}}$, let $T$ be an arbitrary torus over $F$ and let $\widetilde{\mathcal{T}}^{\circ}$ denote the identity component of the Néron-Raynaud model of $T_{K}$ over $\widetilde{U}$. Then $G$ acts on the class group $C_{T, K, S_{K}}=C\left(\widetilde{\mathcal{T}}^{\circ}\right)$. Now, for each prime $v \notin S$, we fix once and for all a prime $w_{v}$ of $K$ lying above $v$ and write $\kappa(v)$ (resp. $\kappa\left(w_{v}\right)$ ) for the residue field of $F$ (resp. $K$ ) at $v\left(\right.$ resp. $w_{v}$ ). Let $G_{w_{v}}$ be the decomposition group of $w_{v}$ in $K / F$. For each $v \notin S$, we will write $d_{v}$ for the dimension of the largest split subtorus of $T_{F_{v}}$,

Received by the editors November 13, 2007.

2000 Mathematics Subject Classification. Primary 20G30; Secondary 11R99.

Key words and phrases. Class numbers of tori, Néron-Raynaud models, Nisnevich cohomology, norm tori.

The author is partially supported by Fondecyt grant 1061209 . 
where $F_{v}$ is the completion of $F$ at $v$. Now let $\Phi_{w_{v}}$ denote the scheme of connected components of the reduction of $\widetilde{\mathcal{T}}$ at $w_{v}$. There exist maps

$$
H^{1}\left(G, T(K) / \widetilde{\mathcal{T}}^{\circ}(\widetilde{U})\right) \rightarrow \bigoplus_{v \notin S} H^{1}\left(G_{w_{v}}, \Phi_{w_{v}}\left(\kappa\left(w_{v}\right)\right)\right)
$$

and

$$
H^{1}\left(G, \widetilde{\mathcal{T}}^{\circ}(\widetilde{U})\right) \rightarrow H^{1}(G, T(K)) .
$$

For the definition of the first map, see the comments following the proof of Lemma 3.1 below. The second map is induced by the canonical inclusion $\widetilde{\mathcal{T}}^{\circ}(\widetilde{U}) \hookrightarrow T(K)$. We denote the kernels of the above maps by $H^{1}\left(G, T(K) / \widetilde{\mathcal{T}}^{\circ}(\widetilde{U})\right)^{\prime}$ and $H^{1}(G, \widetilde{\mathcal{T}} \circ(\widetilde{U}))^{\prime}$, respectively. Finally, let $U=\operatorname{Spec} \mathcal{O}_{F, S}$ and let $\mathcal{T}^{\circ}$ denote the identity component of the Néron-Raynaud model of $T$ over $U$.

Main Theorem. We have

$$
\frac{\left[C_{T, K, S_{K}}^{G}\right]}{\left[C_{T, F, S}\right]}=\frac{\left[\widetilde{\mathcal{T}}^{\circ}(\widetilde{U})^{G}: \mathcal{T}^{\circ}(U)\right]\left[H^{1}\left(G, T(K) / \widetilde{\mathcal{T}}^{\circ}(\widetilde{U})\right)^{\prime}\right]}{\left[H^{1}(G, \widetilde{\mathcal{T}} \circ(\widetilde{U}))^{\prime}\right]} \prod_{v \notin S} e_{v}^{d_{v}} \ell_{v},
$$

where the factors $\ell_{v}$ are certain rational numbers described in Lemma 3.5.

The proof of our Main Theorem is the object of Section 3. In Section 2, after having introduced the necessary notation and terminology, we briefly survey the literature on class groups of tori. Section 4 discusses the particular, but interesting, case of norm tori.

\section{Preliminaries}

Let $F$ be a global field, i.e. $F$ is a finite extension of $\mathbb{Q}$ (the "number field case") or is finitely generated and of transcendence degree 1 over a finite field of constants (the "function field case"). We will write $S_{\infty}$ for the set of archimedean primes of $F$ in the number field case. Let $S$ be a nonempty finite set of primes of $F$ containing $S_{\infty}$ in the number field case and let $\mathcal{O}_{F, S}$ denote the ring of $S$-integers of $F$. We will write $U=\operatorname{Spec} \mathcal{O}_{F, S}$. Further, for any non-archimedean prime $v$ of $F, F_{v}$ will denote the completion of $F$ at $v$ and $\mathcal{O}_{v}$ will denote the ring of integers of $F_{v}$. The residue field of $\mathcal{O}_{v}$ will be denoted by $\kappa(v)$. Further, we will write $j: \operatorname{Spec} F \rightarrow U$ for the inclusion of the generic point of $U$ and, for each closed point $v \in U, i_{v}: \operatorname{Spec} \kappa(v) \rightarrow U$ will denote the similar map at $v$. Note that $i_{v}$ factors through $\operatorname{Spec} \mathcal{O}_{v}$.

Let $K / F$ be a finite Galois extension of global fields with Galois group $G$. We fix a separable algebraic closure $\bar{F}$ of $F$ containing $K$ and write $G_{K}$ for $\operatorname{Gal}(\bar{F} / K)$. Further, we will write $G_{F}$ for $\operatorname{Gal}(\bar{F} / F)$. The set of primes of $K$ lying above the primes in $S$ will be denoted by $S_{K}$. For each prime $v$ of $F$, we fix once and for all a prime $w$ of $K$ lying above $v$ and write $G_{w}$ for the decomposition group of $w$ in $K / F$. When necessary, we will write $w_{v}$ for $w$. The inertia subgroup of $G_{w}$ will be denoted by $I_{w}$. Now, for each $v$ as above, we fix a prime $\bar{w}=\overline{w_{v}}$ of $\bar{F}$ lying above $w=w_{v}$. Then $\bar{F}_{\bar{w}}$ is a separable algebraic closure of $F_{v}$ containing $K_{w}$. We set $I_{\bar{w}}=\operatorname{Gal}\left(\bar{F}_{\bar{w}} / K_{w}^{\mathrm{nr}}\right)$ and $I_{\bar{v}}=\operatorname{Gal}\left(\bar{F}_{\bar{w}} / F_{v}^{\mathrm{nr}}\right)$, where $K_{w}^{\mathrm{nr}}$ (resp. $\left.F_{v}^{\mathrm{nr}}\right)$ is the maximal unramified extension of $K_{w}$ (resp. $F_{v}$ ) inside $\bar{F}_{\bar{w}}$. Clearly, $I_{\bar{w}}$ is a subgroup of $I_{\bar{v}}$ and 
there exist natural isomorphisms $I_{\bar{v}} / I_{\bar{w}}=\operatorname{Gal}\left(K_{w}^{\mathrm{nr}} / F_{v}^{\mathrm{nr}}\right)=I_{w}$. For any subfield $\lambda$ of $\kappa(w)$, we will write $G_{\lambda}$ for $\operatorname{Gal}(\overline{\kappa(w)} / \lambda)$. The quotient $G_{\kappa(v)} / G_{\kappa(w)}$ will be denoted by $G(w)$ (or $G\left(w_{v}\right)$, if necessary) and identified with both $\operatorname{Gal}(\kappa(w) / \kappa(v))$ and $G_{w} / I_{w}$. Further, we will write $e_{v}$ for the ramification index of $v$ in $K$.

Now, for any finite set of primes $S^{\prime}$ of $F$ containing $S$, set

$$
\mathbb{A}_{F, S^{\prime}}=\prod_{v \in S^{\prime}} F_{v} \times \prod_{v \notin S^{\prime}} \mathcal{O}_{v} .
$$

Then the ring of adeles of $F$ is by definition

$$
\mathbb{A}_{F}=\underset{\lim _{S^{\prime} \supset S}}{ } \mathbb{A}_{F, S^{\prime}}
$$

Let $\mathcal{H}$ be a $U$-group scheme of finite type with smooth generic fiber. The class set of $\mathcal{H}$ is by definition the double coset space

$$
C(\mathcal{H})=\mathcal{H}(F) \backslash \mathcal{H}\left(\mathbb{A}_{F}\right) / \mathcal{H}\left(\mathbb{A}_{F, S}\right) .
$$

The cardinality of this set, when finite, is called the class number of $\mathcal{H}$. When $\mathcal{H}_{F}$ is a torus, the class number of $\mathcal{H}$ is well-known to be finite (see 2], Theorem 5.1, for the number field case and [7, Theorem 1.2, Remark 1.3, for the function field case ${ }^{1}$ ). If $\mathcal{H}=\mathbb{G}_{m, U}, C(\mathcal{H})$ may be identified with the $S$-ideal class group of $F$.

Let $\mathcal{O}_{K, S_{K}}$ be the ring of $S_{K}$-integers of $K$, let $\widetilde{U}=\operatorname{Spec} \mathcal{O}_{K, S_{K}}$ and let $\widetilde{\jmath}: \operatorname{Spec} K \rightarrow$ $\widetilde{U}$ be the inclusion of the generic point. Now let $T$ be an $F$-torus and let $\widetilde{\mathcal{T}}$ denote the Néron-Raynaud model of $T_{K}$ over $\widetilde{U}$. Thus $\widetilde{\mathcal{T}}$ is a smooth and separated $\widetilde{U}$ group scheme which is locally of finite type and may be viewed as representing the sheaf $\widetilde{\jmath}_{*} T_{K}$ on the small smooth site over $\widetilde{U}$. See [4, Proposition 10.1.6, p.292. The Néron-Raynaud model of $T$ over $U$ will be denoted by $\mathcal{T}$. Let $\widetilde{\mathcal{T}}^{\circ}\left(\right.$ resp. $\mathcal{T}^{\circ}$ ) denote the (fiberwise) identity component of $\widetilde{\mathcal{T}}$ (resp. $\mathcal{T}$ ). Then $\widetilde{\mathcal{T}}^{\circ}$ is an affine 10, Proposition 3, p.18. ${ }^{2}$ smooth $U$-group scheme of finite type 4], p.290, line 6. For each prime $w \notin S_{K}$, let $\Phi_{w}=i_{w}^{*}\left(\widetilde{\mathcal{T}} / \widetilde{\mathcal{T}}^{\circ}\right)$ denote the sheaf of connected components of $\widetilde{\mathcal{T}}$ at $w$. It is shown in [26, Proposition 2.18, that $\Phi_{w}(\overline{\kappa(w)})$ is a finitely generated $G_{\kappa(w)}$-module. For each prime $v \notin S$, we will write $\Phi_{v}$ for the sheaf of connected components of $\mathcal{T}$ at $v$.

We will need the Nisnevich topology. This is a Grothendieck topology on $U$ which is stronger than the Zariski topology but weaker than the étale topology. It was introduced in 17] in order to generalize the well-known fact that there exists a canonical isomorphism $C_{F, S}=H_{\text {ét }}^{1}\left(U, \mathbb{G}_{m}\right)$, where $C_{F, S}$ is the $S$-ideal class group of $F$. More precisely, if $\mathcal{H}$ is an arbitrary, generically smooth, $U$-group scheme of finite type, then there exists a canonical isomorphism

$$
C(\mathcal{H})=H_{\text {Nis }}^{1}(U, \mathcal{H})
$$

See [18, esp. pp.281-289, for a partial account of the above theory and [17] for full details. In this paper, the $S$-class group $C_{T, F, S}$ of $T$ over $F$ is defined to be $C\left(\mathcal{T}^{\circ}\right)$ (as

\footnotetext{
${ }^{1}$ See [19], §13, pp.280-282, for what appears to be the first published proof of the finiteness of the $S_{\infty}$-class number of a $\mathbb{Q}$-torus $\mathcal{H}_{\mathbb{Q}}$.

2 The proof of this proposition is valid in the function field case as well, as pointed out by D.Lorenzini.
} 
noted above, $\mathcal{T}^{\circ}$ is of finite type and not only generically smooth but in fact smooth). Its cardinality will be denoted by $h_{T, F, S}$.

Remark 2.1. One can also define class groups $C(\mathcal{H})$ for some other integral models of finite type $\mathcal{H}$ of $T$. We choose the model $\mathcal{T}^{\circ}$ because, with this choice, the well-known exact sequence

$$
1 \rightarrow \mathcal{O}_{K, S_{K}}^{*} \rightarrow K^{*} \rightarrow \mathcal{I}_{K, S} \rightarrow C_{K, S} \rightarrow 0
$$

where $\mathcal{I}_{K, S}$ denotes the group of fractional $S$-ideals of $K$, admits the natural generalization (4) below (the exact sequence (4) will play a central role in [9]). Assume now that $F$ is a number field. V.Voskresenskii [24, $\S 20$, and B.Kunyavskii et al. [11, p.47, have defined (for $F$ a number field and $S=S_{\infty}$ ) a class group of $T$ using a certain "standard model" $\mathcal{H}=\mathcal{T}_{0}$ of $T$. Unlike the Néron-Raynaud model of $T$, $\mathcal{T}_{0}$ is of finite type but can be non-smooth. The corresponding class number $h\left(\mathcal{T}_{0}\right)$ is minimal among all class numbers $h\left(\mathcal{T}_{1}\right)$ as $\mathcal{T}_{1}$ runs over the family of all integral models of $T$ of finite type (see [24, p.197). Since $\mathcal{T}^{\circ}$ is such a model (see above), one has $h\left(\mathcal{T}_{0}\right) \mid h_{T, F, S_{\infty}}$ (see [12], p.47, line -3 , for the fact that here we have a divisibility and not only an inequality). When $T$ splits over a tamely ramified extension of $F$, the identity components of $\mathcal{T}$ and $\mathcal{T}_{0}$ are canonically isomorphic [11, Theorem 3, p.49. In this case, therefore, if $\mathcal{T}_{0}$ has connected fibers (which is the case, for example, if $T$ is a quasi-split $F$-torus of the form $T=R_{K / F}\left(\mathbb{G}_{m, K}^{d}\right)$, where $K / F$ is a tamely ramified Galois extension of number fields [11, p.48), then $h\left(\mathcal{T}_{0}\right)$ and $h_{T, F, S_{\infty}}$ coincide. We should also mention here the work of M.Morishita [15] who, generalizing work of T.Ono, obtained a formula for the class number of the natural model

$$
R_{\mathcal{O}_{K, S_{K}} / \mathcal{O}_{F, S}}^{(1)}\left(\mathbb{G}_{m, \mathcal{O}_{K, S_{K}}}\right)=\operatorname{Ker}\left[R_{\mathcal{O}_{K, S_{K}} / \mathcal{O}_{F, S}}\left(\mathbb{G}_{m, \mathcal{O}_{K, S_{K}}}\right) \stackrel{N_{\mathcal{O}}}{\longrightarrow} \mathbb{G}_{m, \mathcal{O}_{F, S}}\right]
$$

of the norm torus $R_{K / F}^{(1)}\left(\mathbb{G}_{m, K}\right)$. See Section 4 for a discussion of norm tori in the context of this paper (we hope to discuss the connections between the approach of Section 4 and that of [15] in a subsequent publication. At this time we note that, in general, it is quite difficult to compare the arithmetic invariants (e.g., class numbers) associated to a given model of a torus with the corresponding invariants associated to another model).

We will write $X=\operatorname{Hom}\left(T(\bar{F}), \bar{F}^{*}\right)$ for the $G_{F}$-module of characters of $T$. For any $G_{F}$-lattice $Y$, we will write $Y^{\vee}$ for the $\mathbb{Z}$-linear dual of $Y$, i.e., $Y^{\vee}=\operatorname{Hom}_{\mathbb{Z}}(Y, \mathbb{Z})$.

If $f: A \rightarrow B$ is a homomorphism of abelian groups with finite kernel and cokernel, we define

$$
q(f)=\frac{[\operatorname{Coker} f]}{[\operatorname{Ker} f]} .
$$

This function is multiplicative on short exact sequences, in the sense that if $f^{\bullet}: X^{\bullet} \rightarrow$ $Y^{\bullet}$ is a map of short exact sequences and $q\left(f^{r}\right)$ is defined for $r=1,2,3$, then $q\left(f^{2}\right)=$ $q\left(f^{1}\right) q\left(f^{3}\right)$.

If $M$ is any abelian group and $m$ is any positive integer, $M_{m}$ will denote its $m$ torsion subgroup and we will write $M / m$ for $M / m M$. Applying $\operatorname{Tor}^{\mathbb{Z}}(\mathbb{Z} / m,-)$ to a given exact sequence $0 \rightarrow A \rightarrow B \rightarrow C \rightarrow 0$ of abelian groups, we obtain an exact sequence

$$
0 \rightarrow A_{m} \rightarrow B_{m} \rightarrow C_{m} \stackrel{c}{\longrightarrow} A / m \rightarrow B / m \rightarrow C / m \rightarrow 0
$$


where $c$ is a certain "connecting homomorphism". See, e.g., 25], §3.1.1, p.66. The dual of $M$ is by definition $M^{D}=\operatorname{Hom}(M, \mathbb{Q} / \mathbb{Z})$. If $G$ is a group and $M$ is a (left) $G$ module, $M^{D}$ will be endowed with its natural $G$-action (see, e.g., 1], p.94). Further, $M_{G}$ (resp. $M^{G}$ ) will denote the largest quotient (resp. subgroup) of $M$ on which $G$ acts trivially. It is not difficult to check that $\left(M^{D}\right)^{G}=\left(M_{G}\right)^{D}$. Further, if $M$ is finite and $G$ is pro-cyclic, then $\left[M_{G}\right]=\left[M^{G}\right]$ (see, e.g., [1, proof of Proposition 11, p.109). It follows that $\left[\left(M^{D}\right)^{G}\right]=\left[M^{G}\right]$ (we will use the latter fact often when referring to 26], Corollary 2.18, p.175). We also note that, if $H$ is a normal subgroup of $G$, then there exists a canonical isomorphism $\mathbb{Z}[G]^{H}=\mathbb{Z}[G / H]$. In addition to the above, we will need the following well-known facts: if $M=\mathbb{Z}[G] \otimes Y$ for some abelian group $Y^{3}$, then the Tate groups $\widehat{H}_{0}(G, M)=\widehat{H}^{0}(G, M)=0$ (see, e.g., 11, Proposition 6, p.102). If $Y$ is a torsion-free abelian group, then $\operatorname{Tor}_{1}^{\mathbb{Z}}(Y,-)=0$. Further, if $G$ is a torsion group which acts trivially on $Y$, then $H^{1}(G, Y)=\operatorname{Hom}(G, Y)=0$.

\section{Proof of the main theorem}

There exists a natural exact sequence of sheaves on $\widetilde{U}_{\mathrm{sm}}$ :

$$
0 \rightarrow \widetilde{\mathcal{T}}^{\circ} \rightarrow \widetilde{\mathcal{T}} \rightarrow \bigoplus_{w \notin S_{K}}\left(i_{w}\right)_{*} \Phi_{w} \rightarrow 0 .
$$

See 22, Lemma 10.1.1, p.157. The preceding exact sequence induces an exact sequence

$$
1 \rightarrow \widetilde{\mathcal{T}}^{\circ}(\widetilde{U}) \rightarrow T(K) \rightarrow \bigoplus_{w \notin S_{K}} \Phi_{w}(\kappa(w)) \rightarrow H_{\text {êt }}^{1}\left(\widetilde{U}, \widetilde{\mathcal{T}}^{\circ}\right) \rightarrow H_{\text {èt }}^{1}(\widetilde{U}, \widetilde{\mathcal{T}}) .
$$

Lemma 3.1. There exists a canonical isomorphism

$$
\operatorname{Ker}\left[H_{\text {ét }}^{1}\left(\widetilde{U}, \widetilde{\mathcal{T}}^{\circ}\right) \rightarrow H_{\text {ét }}^{1}(\widetilde{U}, \widetilde{\mathcal{T}})\right]=C_{T, K, S_{K}} .
$$

Proof. Since $\widetilde{\mathcal{T}}=\widetilde{\jmath}_{*} T_{K}$ as étale sheaves, the Cartan-Leray spectral sequence

$$
H_{\text {ét }}^{p}\left(\widetilde{U}, R^{q} \widetilde{\jmath}_{*} T_{K}\right) \Longrightarrow H_{\text {ét }}^{p+q}(K, T)
$$

yields an injection $H_{\text {êt }}^{1}(\widetilde{U}, \widetilde{\mathcal{T}})=H_{\text {ét }}^{1}\left(\widetilde{U}, \widetilde{\jmath}_{*} T_{K}\right) \hookrightarrow H_{\text {ét }}^{1}(K, T)$. Thus $H_{\text {ét }}^{1}(\widetilde{U}, \widetilde{\mathcal{T}})$ may be replaced with $H^{1}(K, T)$ in the statement of the lemma. On the other hand, [18, 1.44.2, p.286, shows that there exists a canonical isomorphism

$$
\operatorname{Ker}\left[H_{\text {ét }}^{1}\left(\widetilde{U}, \widetilde{\mathcal{T}}^{\circ}\right) \rightarrow H^{1}(K, T)\right]=H_{\text {Nis }}^{1}\left(\widetilde{U}, \widetilde{\mathcal{T}}^{\circ}\right) .
$$

Composing the above isomorphism with the isomorphism (1), with obtain the desired result.

The above lemma and (3) show that there exists a canonical exact sequence of abelian groups

$$
1 \rightarrow \widetilde{\mathcal{T}}^{\circ}(\widetilde{U}) \rightarrow T(K) \rightarrow \bigoplus_{v \notin S} \bigoplus_{w \mid v} \Phi_{w}(\kappa(w)) \rightarrow C_{T, K, S_{K}} \rightarrow 0 .
$$

We will show that (4) is in fact an exact sequence of $G$-modules. Let $v \notin S$ and let $w$ be any prime of $K$ lying above $v$. There exists a canonical action of $G_{w}$ on

\footnotetext{
${ }^{3}$ All tensor products in this paper are taken over $\mathbb{Z}$.
} 
$T_{K_{w}}=T \times_{\operatorname{Spec} F} \operatorname{Spec} K_{w}$ which extends the natural action of $G_{w}$ on $\operatorname{Spec} K_{w}$. Now, since $\widetilde{\mathcal{T}} \times_{\widetilde{U}} \operatorname{Spec} \mathcal{O}_{w}$ is a Néron-Raynaud model of $T_{K_{w}}$ over $\operatorname{Spec} \mathcal{O}_{w}$ 4, p.290, line 3, the Néron mapping property [4], Definition 1.2.1, p.12, shows that the action of $G_{w}$ on $T_{K_{w}}$ extends uniquely to an action of $G_{w}$ on $\widetilde{\mathcal{T}} \times_{\widetilde{U}} \operatorname{Spec} \mathcal{O}_{w}$. The latter induces an action of $G_{w}$ on $\left(i_{w}\right)_{*} \Phi_{w}$ such that the subgroup $I_{w} \subset G_{w}$ acts trivially. It follows that $\left(i_{w}\right)_{*} \Phi_{w}$ is canonically endowed with a $G(w)$-action. In particular, $G(w)$ acts on $\Phi_{w}(\kappa(w))$. Now there exists a natural action of $G$ on $\bigoplus_{w \mid v} \Phi_{w}(\kappa(w))$ which identifies the latter group with the $G$-module coinduced by the $G_{w_{v}}$-module $\Phi_{w_{v}}\left(\kappa\left(w_{v}\right)\right)$, where $w_{v}$ is the prime of $K$ lying above $v$ fixed previously. Note that, by Shapiro's lemma,

$$
H^{i}\left(G, \bigoplus_{w \mid v} \Phi_{w}(\kappa(w))\right)=H^{i}\left(G_{w_{v}}, \Phi_{w_{v}}\left(\kappa\left(w_{v}\right)\right)\right)
$$

for every $i \geq 0$. Note also that, since $I_{w_{v}}$ acts trivially on $\Phi_{w_{v}}\left(\kappa\left(w_{v}\right)\right)$,

$$
\Phi_{w_{v}}\left(\kappa\left(w_{v}\right)\right)^{G w_{v}}=\Phi_{w_{v}}\left(\kappa\left(w_{v}\right)\right)^{G\left(w_{v}\right)} .
$$

Similarly, the Néron mapping property implies that $\widetilde{\mathcal{T}}^{\circ}(\widetilde{U})$ is canonically endowed with a $G$-action. It is now not difficult to see, using the functoriality properties of the Néron-Raynaud model, that (4) is indeed an exact sequence of $G$-modules.

We now observe that (4) induces an exact sequence

$$
C_{T, K, S_{K}}^{G} \stackrel{\alpha}{\longrightarrow} H^{1}\left(G, T(K) / \widetilde{\mathcal{T}}^{\circ}(\widetilde{U})\right) \stackrel{\beta}{\longrightarrow} \bigoplus_{v \notin S} H^{1}\left(G_{w_{v}}, \Phi_{w_{v}}\left(\kappa\left(w_{v}\right)\right)\right) .
$$

Define

$$
\left(C_{T, K, S_{K}}^{G}\right)_{\operatorname{trans}}=\operatorname{Ker}(\alpha)
$$

and

$$
H^{1}\left(G, T(K) / \widetilde{\mathcal{T}}^{\circ}(\widetilde{U})\right)^{\prime}=\operatorname{Ker}(\beta) .
$$

Then

$$
\left[C_{T, K, S_{K}}^{G}\right]=\left[\left(C_{T, K, S_{K}}^{G}\right)_{\operatorname{trans}}\right]\left[H^{1}\left(G, T(K) / \widetilde{\mathcal{T}}^{\circ}(\widetilde{U})\right)^{\prime}\right] .
$$

Remark 3.2. If $K$ splits $T$, i.e., $T_{K}=\mathbb{G}_{m, K}^{d}$ for some $d$, then $H^{1}\left(G_{w_{v}}, \Phi_{w_{v}}\left(\kappa\left(w_{v}\right)\right)\right)=$ $\operatorname{Hom}\left(G_{w_{v}}, \mathbb{Z}^{d}\right)=0$. In this case, therefore,

$$
H^{1}\left(G, T(K) / \widetilde{\mathcal{T}}^{\circ}(\widetilde{U})\right)^{\prime}=H^{1}\left(G, T(K) / \widetilde{\mathcal{T}}^{\circ}(\widetilde{U})\right) .
$$

Now let $\pi: \widetilde{U} \rightarrow U$ be the canonical map and let $v \notin S$. The base change morphism $\pi^{*} \mathcal{T}=\mathcal{T} \times_{U} \widetilde{U} \rightarrow \widetilde{\mathcal{T}}$ [4, $\S 7.2$, Theorem 1(i), p.176, induces a canonical map $\pi^{*}\left(i_{v}\right)_{*} \Phi_{v} \rightarrow \bigoplus_{w \mid v}\left(i_{w}\right)_{*} \Phi_{w}$. Taking $\widetilde{U}$-sections and noting that $G$ acts trivially on $\Phi_{v}(\kappa(v))$, we obtain a canonical capitulation map

$$
\delta_{v}: \Phi_{v}(\kappa(v)) \rightarrow\left(\bigoplus_{w \mid v} \Phi_{w}(\kappa(w))\right)^{G}=\Phi_{w_{v}}\left(\kappa\left(w_{v}\right)\right)^{G\left(w_{v}\right)}
$$

On the other hand, identifying $C_{T, F, S}$ and $C_{T, K, S_{K}}$ with $H_{\mathrm{Nis}}^{1}\left(U, \mathcal{T}^{\circ}\right)$ and $H_{\mathrm{Nis}}^{1}\left(\widetilde{U}, \widetilde{\mathcal{T}}^{\circ}\right)$ (respectively) via (1), we may define a canonical capitulation map

$$
j_{T, K / F}: C_{T, F, S} \rightarrow C_{T, K, S_{K}}^{G}
$$

as the composite

$$
H_{\mathrm{Nis}}^{1}\left(U, \mathcal{T}^{\circ}\right) \stackrel{\mathrm{ad}}{\rightarrow} H_{\mathrm{Nis}}^{1}\left(U, \pi_{*} \pi^{*} \mathcal{T}^{\circ}\right) \hookrightarrow H_{\mathrm{Nis}}^{1}\left(\widetilde{U}, \pi^{*} \mathcal{T}^{\circ}\right)^{G} \stackrel{\mathrm{bc}}{\rightarrow} H_{\mathrm{Nis}}^{1}\left(\widetilde{U}, \widetilde{\mathcal{T}}^{\circ}\right)^{G},
$$


where "ad" is induced by the adjoint morphism $\mathcal{T}^{\circ} \rightarrow \pi_{*} \pi^{*} \mathcal{T}^{\circ}$, the middle injection is the first nontrivial map in the exact sequence of terms of low degree belonging to the Cartan-Leray spectral sequence $H_{\mathrm{Nis}}^{p}\left(U, R^{q} \pi_{*} \pi^{*} \mathcal{T}^{\circ}\right) \Longrightarrow H_{\mathrm{Nis}}^{p+q}\left(\widetilde{U}, \pi^{*} \mathcal{T}^{\circ}\right)$ [18, 1.22.1, p.270, and the map "bc" is induced by the base change morphism $\pi^{*} \mathcal{T}^{\circ} \rightarrow \widetilde{\mathcal{T}}^{\circ}$. It is not difficult to check that the image of $j_{T, K / F}$ is contained in $\left(C_{T, K, S_{K}}^{G}\right)_{\text {trans }}$. We will write $j_{T, K / F}^{\prime}: C_{T, F, S} \rightarrow\left(C_{T, K, S_{K}}^{G}\right)_{\text {trans }}$ for the induced map. Then (6) may be rewritten as

$$
\frac{\left[C_{T, K, S_{K}}^{G}\right]}{\left[C_{T, F, S}\right]}=q\left(j_{T, K / F}^{\prime}\right)\left[H^{1}\left(G, T(K) / \widetilde{\mathcal{T}}^{\circ}(\widetilde{U})\right)^{\prime}\right] .
$$

Now there exists a natural exact commutative diagram

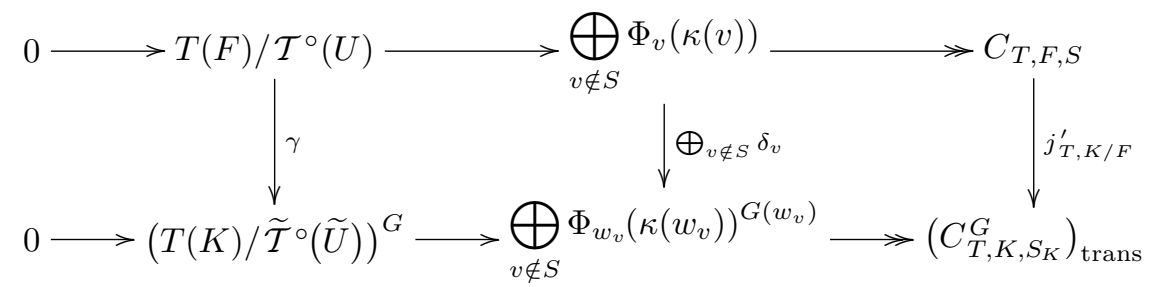

where, for each $v \notin S, \delta_{v}$ is the capitulation map (7) and $\gamma$ is induced by $\bigoplus_{v \notin S} \delta_{v}$.

Lemma 3.3. For each $v \notin S$, there exists an isomorphism of finite groups

$$
\operatorname{Ker}\left[\Phi_{v}(\kappa(v)) \stackrel{\delta_{v}}{\longrightarrow} \Phi_{w_{v}}\left(\kappa\left(w_{v}\right)\right)^{G\left(w_{v}\right)}\right]=H^{1}\left(I_{w_{v}}, T\left(K_{w_{v}}^{\mathrm{nr}}\right)\right)^{G_{\kappa(v)}} .
$$

Here $I_{w_{v}}$ is identified with $\operatorname{Gal}\left(K_{w_{v}}^{n r} / F_{v}^{n r}\right)$. In particular, if $v$ is unramified in $K / F$, then $\delta_{v}$ is injective.

Proof. Set $w=w_{v}$. Since $\operatorname{Ker} \delta_{v}$ is a torsion group (cf. [8], Theorem 1), we have $\operatorname{Ker} \delta_{v}=\operatorname{Ker} \delta_{v}^{\prime}$, where $\delta_{v}^{\prime}: \Phi_{v}(k(v))_{\text {tors }} \rightarrow \Phi_{w}(\kappa(w))_{\text {tors }}^{G(w)}$ is the induced map. Since $\Phi_{v}(k(v))_{\text {tors }}$ is finite by 26, Corollary 2.18, we conclude that $\operatorname{Ker} \delta_{v}$ is finite as well. Now, by [26], Corollary 2.18, and [14], Corollary I.2.4, p.35, there exists a natural exact commutative diagram

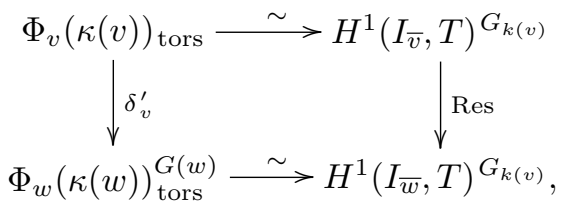

where the right-hand vertical map is induced by the restriction map $H^{1}\left(I_{\bar{v}}, T\right) \rightarrow$ $H^{1}\left(I_{\bar{w}}, T\right)$. The commutativity of the above diagram follows by examining the proofs of Lemma 2.13, Proposition 2.14, Lemma 2.17 and Corollary 2.18 in [26]. The key point is that the map $H^{1}\left(I_{\bar{w}}, X\right) \rightarrow H^{1}\left(I_{\bar{v}}, X\right)$ (notation as in [op.cit.]) which corresponds to the map $\operatorname{Ext}_{\mathbb{Z}}^{1}\left(\Phi_{w}, \mathbb{Z}\right) \rightarrow \operatorname{Ext}_{\mathbb{Z}}^{1}\left(\Phi_{v}, \mathbb{Z}\right)$ under the isomorphism of [op.cit., Proposition 2.14] is induced by the norm map $N_{I_{w}}: X^{I_{\bar{w}}} \rightarrow X^{I_{\bar{v}}}$ (see, especially, the diagram at the bottom of p.174 of [op.cit.]). The result is now immediate from the inflation-restriction exact sequence. 
An $F$-torus $T$ is said to have multiplicative reduction at a nonarchimedean prime $v$ of $F$ if $\mathcal{T}_{\mathcal{O}_{v}}^{\circ}$ is a torus over $\mathcal{O}_{v}$. The following are equivalent conditions (see [16], (1.1), p.462): (a) $T$ has multiplicative reduction at $v$ as defined above; (b) $\mathcal{T}_{\kappa(v)}^{\circ}$ is a torus over $\kappa(v)$; (c) $I_{\bar{v}}$ acts trivially on $X$; (d) $T$ splits over an unramified extension of $F_{v}$. Since only finitely many primes of $F$ can ramify in a splitting field of $T$, we conclude that $T$ has multiplicative reduction at all but finitely many primes of $F$.

Lemma 3.4. Let $v$ be a nonarchimedean prime of $F$ which is unramified in $K / F$ and where $T$ has multiplicative reduction. Then the capitulation map

$$
\delta_{v}: \Phi_{v}(\kappa(v)) \rightarrow \Phi_{w_{v}}\left(\kappa\left(w_{v}\right)\right)^{G\left(w_{v}\right)}
$$

is an isomorphism.

Proof. Let $T_{v}^{*}$ be the largest split subtorus of $T_{F_{v}}$ and let $d_{v}$ be its dimension. Let $\Phi_{v}^{*}$ (resp. $\Phi_{w_{v}}^{*}$ ) denote the sheaf of connected components of the Néron-Raynaud model of $T_{v}^{*}$ (resp. $\left(T_{v}^{*}\right)_{K_{w_{v}}}$ ). By Proposition 3.2 of [3], the Néron mapping property implies the existence of an isomorphism $\Phi_{w_{v}}^{*}\left(\kappa\left(w_{v}\right)\right) \simeq \Phi_{w_{v}}\left(\kappa\left(w_{v}\right)\right)$. Now, by the definition of the $G\left(w_{v}\right)$-action on both of these groups (see above) and the functoriality properties of the Néron-Raynaud model, the latter is in fact an isomorphism of $G\left(w_{v}\right)$-modules. We conclude that there exists a commutative diagram

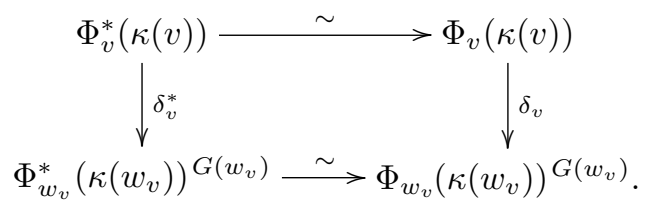

The left-hand vertical map $\delta_{v}^{*}$ may be identified with the multiplication-by- $e_{v}$ map $\mathbb{Z}^{d_{v}} \rightarrow \mathbb{Z}^{d_{v}}$. Since $e_{v}=1$ by hypothesis, the lemma follows.

Lemmas 3.3 and 3.4 show that the map $\bigoplus_{v \notin S} \delta_{v}$ in diagram (9) has a finite kernel and cokernel. Therefore

$$
q\left(\oplus_{v \notin S} \delta_{v}\right)=\prod_{v \notin S} q\left(\delta_{v}\right)
$$

is defined and diagram (9) yields

$$
\prod_{v \notin S} q\left(\delta_{v}\right)=q(\gamma) q\left(j_{T, K / F}^{\prime}\right) .
$$

Consequently, (8) yields the identity

$$
\frac{\left[C_{T, K, S_{K}}^{G}\right]}{\left[C_{T, F, S}\right]}=\frac{\left[H^{1}\left(G, T(K) / \widetilde{\mathcal{T}}^{\circ}(\widetilde{U})\right)^{\prime}\right]}{q(\gamma)} \cdot \prod_{v \notin S} q\left(\delta_{v}\right) .
$$

It remains to compute the factors $q\left(\delta_{v}\right)$ (for $v \notin S$ ) and $q(\gamma)$. We compute first the local factors $q\left(\delta_{v}\right)$.

Let $v \notin S$. The inertia group $I_{\bar{v}}$ acts on $X$ through a finite quotient $J_{\bar{v}}$ (say), and we have a natural map

$$
N_{v}: X \rightarrow X^{I_{\bar{v}}}, \chi \mapsto \sum_{g \in J_{\bar{v}}} \chi^{g} .
$$


Let $\widehat{T}_{v}$ be the $F_{v}$-torus corresponding to the subgroup $\operatorname{Ker} N_{v}$ of $X$ and let $T_{v}^{*}$ be the largest split subtorus of $T_{F_{v}}$. Let $d_{v}$ be the dimension of $T_{v}^{*}$. Set $w=w_{v}$. Since $H^{1}(G(w), \mathbb{Z})=\operatorname{Hom}(G(w), \mathbb{Z})=0$, Corollary 3.3 of [3] yields an exact commutative diagram

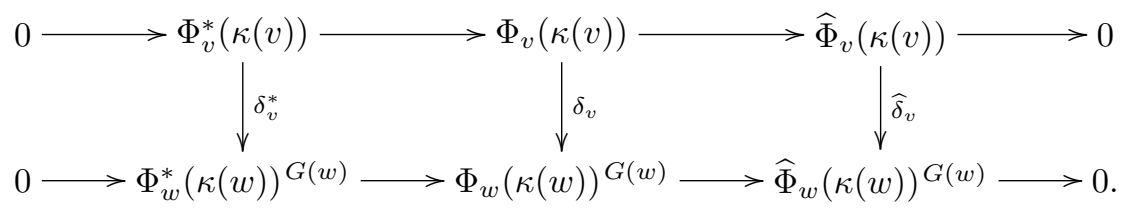

Since the left-hand vertical map $\delta_{v}^{*}$ can be identified with the multiplication-by- $e_{v}$ $\operatorname{map} \mathbb{Z}^{d_{v}} \rightarrow \mathbb{Z}^{d_{v}}$, we have

$$
q\left(\delta_{v}^{*}\right)=e_{v}^{d_{v}} .
$$

On the other hand, by [26, Corollary 2.19(b), p.175, and the fact that $\left[M_{G_{\kappa(v)}}\right]=$ $\left[M^{G_{\kappa(v)}}\right]$ for any finite $G_{\kappa(v)}$-module $M, \widehat{\Phi}_{v}(\overline{\kappa(v)})$ and $\widehat{\Phi}_{w}(\overline{\kappa(w)})$ are finite $G_{\kappa(v)^{-}}$ modules and

$$
\begin{aligned}
q\left(\widehat{\delta}_{v}\right) & =\left[\widehat{\Phi}_{w}(\overline{\kappa(w)})^{G_{\kappa(v)}}\right] /\left[\widehat{\Phi}_{v}(\overline{\kappa(v)})^{G_{\kappa(v)}}\right] \\
& =\left[H^{1}\left(I_{\bar{w}}, \operatorname{Ker} N_{v}\right)^{G_{\kappa(v)}}\right] /\left[H^{1}\left(I_{\bar{v}}, \operatorname{Ker} N_{v}\right)^{G_{\kappa(v)}}\right] .
\end{aligned}
$$

Thus the following holds.

Lemma 3.5. Let $v \notin S$. Then

$$
q\left(\delta_{v}\right)=e_{v}^{d_{v}} \frac{\left[H^{1}\left(I_{\bar{w}_{v}}, \operatorname{Ker} N_{v}\right)^{G_{\kappa(v)}}\right]}{\left[H^{1}\left(I_{\bar{v}}, \operatorname{Ker} N_{v}\right)^{G_{\kappa(v)}}\right]} .
$$

The factor $\ell_{v}=\left[H^{1}\left(I_{\bar{w}_{v}}, \operatorname{Ker} N_{v}\right)^{G_{\kappa(v)}}\right] /\left[H^{1}\left(I_{\bar{v}}, \operatorname{Ker} N_{v}\right)^{G_{\kappa(v)}}\right]$ is a rational number.

Remark 3.6. For $v \notin S$, set $w=w_{v}$. If $T$ splits over $K$ (or, more generally, if $T_{K}$ has multiplicative reduction at $w$ so that $I_{\bar{w}}$ acts trivially on $X$ ), then

$$
H^{1}\left(I_{\bar{w}}, \operatorname{Ker} N_{v}\right)=\operatorname{Hom}\left(I_{\bar{w}}, \operatorname{Ker} N_{v}\right)=0
$$

since $I_{\bar{w}}$ is torsion and Ker $N_{v}$ is a torsion-free abelian group with trivial $I_{\bar{w}}$-action. It now follows from the inflation-restriction exact sequence that $H^{1}\left(I_{\bar{v}}, \operatorname{Ker} N_{v}\right)=$ $H^{1}\left(I_{w}, \operatorname{Ker} N_{v}\right)$. Further, $G_{k(v)}$ acts on this group through the quotient $G(w)=$ $G_{k(v)} / G_{k(w)}$. Thus, in this case, the formula of the lemma has the simpler form $q\left(\delta_{v}\right)=e_{v}^{d_{v}} /\left[H^{1}\left(I_{w_{v}}, \operatorname{Ker} N_{v}\right)^{G\left(w_{v}\right)}\right]$.

Now set

$$
H^{1}\left(G, \widetilde{\mathcal{T}}^{\circ}(\widetilde{U})\right)^{\prime}=\operatorname{Ker}\left[H^{1}\left(G, \widetilde{\mathcal{T}}^{\circ}(\widetilde{U})\right) \rightarrow H^{1}(G, T(K))\right],
$$

where the map involved is induced by the natural map $\widetilde{\mathcal{T}}^{\circ}(\widetilde{U}) \rightarrow T(K)$. Further, recall the map

$$
\gamma: T(F) / \mathcal{T}^{\circ}(U) \rightarrow\left(T(K) / \widetilde{\mathcal{T}}^{\circ}(\widetilde{U})\right)^{G}
$$

from diagram (9). 
Lemma 3.7. We have

$$
q(\gamma)=\frac{\left[H^{1}(G, \widetilde{\mathcal{T}} \circ(\widetilde{U}))^{\prime}\right]}{\left[\widetilde{\mathcal{T}} \circ(\widetilde{U})^{G}: \mathcal{T}^{\circ}(U)\right]} .
$$

Proof. The commutativity of the exact diagram

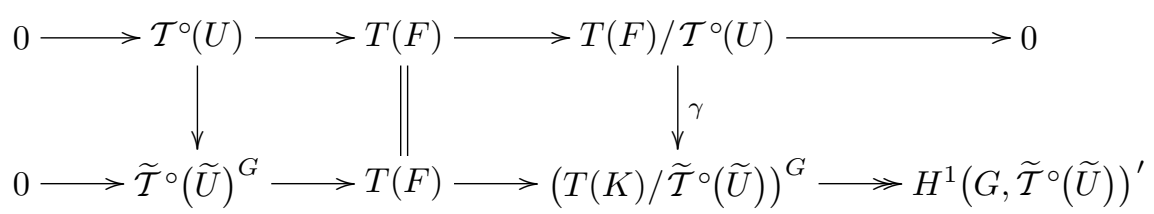

yields canonical isomorphisms

$$
\operatorname{Ker}(\gamma)=\widetilde{\mathcal{T}}^{\circ}(\widetilde{U})^{G} / \mathcal{T}^{\circ}(U)
$$

and

$$
\operatorname{Coker}(\gamma)=H^{1}\left(G, \widetilde{\mathcal{T}}^{\circ}(\widetilde{U})\right)^{\prime}
$$

The lemma is now clear.

We can now state our generalization of Chevalley's ambiguous class number formula.

Theorem 3.8. We have

$$
\frac{\left[C_{T, K, S_{K}}^{G}\right]}{\left[C_{T, F, S}\right]}=\frac{\left[\widetilde{\mathcal{T}}^{\circ}(\widetilde{U})^{G}: \mathcal{T}^{\circ}(U)\right]\left[H^{1}\left(G, T(K) / \widetilde{\mathcal{T}}^{\circ}(\widetilde{U})\right)^{\prime}\right]}{\left[H^{1}(G, \widetilde{\mathcal{T}} \circ(\widetilde{U}))^{\prime}\right]} \prod_{v \notin S} q\left(\delta_{v}\right),
$$

where $H^{1}\left(G, T(K) / \widetilde{\mathcal{T}}^{\circ}(\widetilde{U})\right)^{\prime}$ and $H^{1}\left(G, \widetilde{\mathcal{T}}^{\circ}(\widetilde{U})\right)^{\prime}$ are given by (5) and (11), respectively, and, for each $v \notin S, q\left(\delta_{v}\right)$ is given by Lemma 3.5.

Proof. This is immediate from (10) and Lemma 3.7.

The next formula is closer to Chevalley's original formula than that of the theorem 4

Corollary 3.9. Assume that $T_{K}$ has multiplicative reduction over $\widetilde{U}$, i.e., that $S_{K}$ contains all primes of bad reduction for $T_{K}$. Then

$$
\frac{\left[C_{K, T, S_{K}}^{G}\right]}{\left[C_{T, F, S}\right]}=\frac{\left[H^{1}\left(G,\left(X^{G_{K}}\right)^{\vee} \otimes\left(K^{*} / \mathcal{O}_{K, S_{K}}^{*}\right)\right)^{\prime}\right] \prod_{v \notin S} q\left(\delta_{v}\right)}{\left[H^{1}\left(G,\left(X^{G_{K}}\right)^{\vee} \otimes \mathcal{O}_{K, S_{K}}^{*}\right)^{\prime}\right]\left[\left(\left(X^{G_{K}}\right)^{\vee} \otimes \mathcal{O}_{K, S_{K}}^{*}\right)^{G}: \mathcal{T}^{\circ}(U)\right]^{-1}} .
$$

\footnotetext{
${ }^{4}$ We write $\left[\left(\left(X^{G_{K}}\right)^{\vee} \otimes \mathcal{O}_{K, S_{K}}^{*}\right)^{G}: \mathcal{T}^{\circ}(U)\right]$ in the denominator with an exponent -1 in order to make the formula fit on one line.
} 
Proof. By hypothesis, $\widetilde{\mathcal{T}}^{\circ}$ is a torus over $\widetilde{U}$, whence

$$
\widetilde{\mathcal{T}}^{\circ}=\mathcal{H o m}_{\widetilde{U}}\left(X\left(\widetilde{\mathcal{T}}^{\circ}\right), \mathbb{G}_{m}\right)
$$

as sheaves on $\widetilde{U}_{\text {ét }}$, where $X\left(\widetilde{\mathcal{T}}^{\circ}\right)$ is the sheaf of characters of $\widetilde{\mathcal{T}}^{\circ}$. Now, since $X\left(\widetilde{\mathcal{T}}^{\circ}\right)$ is an étale-locally constant $\mathbb{Z}$-constructible sheaf on $\widetilde{U}, X\left(\widetilde{\mathcal{T}}^{\circ}\right)(\widetilde{U})=X^{G_{K}}$ (see, e.g., [13, p.156). Consequently,

$$
\widetilde{\mathcal{T}}^{\circ}(\widetilde{U})=\operatorname{Hom}\left(X^{G_{K}}, \mathcal{O}_{K, S_{K}}^{*}\right)=\left(X^{G_{K}}\right)^{\vee} \otimes \mathcal{O}_{K, S_{K}}^{*} .
$$

The corollary is now immediate.

Remark 3.10. The corollary is valid for any set $S$ verifying our general assumptions if $T$ splits over $K$. In this case $X^{G_{K}}=X$ and, using Remark 3.2, the formula of the corollary has the following simpler form:

$$
\frac{\left[C_{K, T, S_{K}}^{G}\right]}{\left[C_{T, F, S}\right]}=\frac{\left[H^{1}\left(G, X^{\vee} \otimes\left(K^{*} / \mathcal{O}_{K, S_{K}}^{*}\right)\right)\right] \prod_{v \notin S} q\left(\delta_{v}\right)}{\left[H^{1}\left(G, X^{\vee} \otimes \mathcal{O}_{K, S_{K}}^{*}\right)^{\prime}\right]\left[\left(\left(X^{\vee} \otimes \mathcal{O}_{K, S_{K}}^{*}\right)^{G}: \mathcal{T}^{\circ}(U)\right]^{-1}\right.} .
$$

\section{Norm tori}

Define a $G_{F}$-module $X$ by the exactness of the sequence

$$
0 \rightarrow \mathbb{Z} \stackrel{\varepsilon}{\longrightarrow} \mathbb{Z}[G] \rightarrow X \rightarrow 0,
$$

where $\varepsilon$ is given by $\varepsilon(1)=\sum_{\sigma \in G} \sigma$. This exact sequence induces an exact sequence of $F$-tori

$$
0 \rightarrow T \rightarrow R_{K / F}\left(\mathbb{G}_{m, K}\right) \stackrel{N}{\longrightarrow} \mathbb{G}_{m, F} \rightarrow 0,
$$

where $R_{K / F}\left(\mathbb{G}_{m, K}\right)$ is the Weil restriction of $\mathbb{G}_{m, K}, N$ is induced by the norm map $K^{*} \rightarrow F^{*}$ and $T=R_{K / F}^{(1)}\left(\mathbb{G}_{m, K}\right)$ is the kernel of $N$. Note that $T_{K} \simeq \mathbb{G}_{m, K}^{n-1}$, where $n=[K: F]$. For any $v \notin S$, let $w=w_{v}$ be the prime of $K$ lying above $v$ fixed previously. We will write $f_{v}$ for the residue class degree $[\kappa(w): \kappa(v)]$ and $G_{w}^{\text {ab }}$ for the largest abelian quotient of $G_{w}$, i.e., $G_{w}^{\mathrm{ab}}=G_{w} / G_{w}^{\prime}$, where $G_{w}^{\prime}$ is the commutator subgroup of $G_{w}$. Note that $G(w)$ is a cyclic group of order $f_{v}$ and $\left[G_{w}\right]=e_{v} f_{v}$. Further, since $G_{w} / I_{w}=G(w)$ is abelian, $I_{w}$ contains $G_{w}^{\prime}$.

The dimension $d_{v}$ of the largest split subtorus of $T_{F_{v}}$ is the rank of $X^{G_{w}}$. Since $H^{1}\left(G_{w}, \mathbb{Z}\right)=0$ and $\mathbb{Z}[G]$ is a free (right) $\mathbb{Z}\left[G_{w}\right]$-module of rank $\left[G: G_{w}\right],(12)$ shows that $d_{v}=\left[G: G_{w}\right]-1$. We will now compute $q\left(\delta_{v}\right)=e_{v}^{d_{v}} /\left[H^{1}\left(I_{w}, \operatorname{Ker} N_{v}\right)^{G(w)}\right]$ (see Remark 3.6). Recall the norm element $N_{I_{w}}=\sum_{\tau \in I_{w}} \tau \in \mathbb{Z}[G]$. Since $\mathbb{Z}[G]=$ $\mathbb{Z}\left[I_{w}\right]^{\left[G: I_{w}\right]}$, the multiplication-by- $N_{I_{w}}$ map $\mathbb{Z}[G] \rightarrow \mathbb{Z}[G]^{I_{w}}$ is surjective. Further, since $H^{1}\left(I_{w}, \mathbb{Z}\right)=0$, the canonical map $\mathbb{Z}[G]^{I_{w}} \rightarrow X^{I_{w}}$ is surjective as well. It follows that $N_{v}: X \rightarrow X^{I_{w}}$ is surjective. Consequently, we have a natural exact sequence of $I_{w}$-modules

$$
0 \rightarrow \operatorname{Ker} N_{v} \rightarrow X \stackrel{N_{v}}{\longrightarrow} X^{I_{w}} \rightarrow 0 .
$$

Taking $I_{w}$-cohomology of the above exact sequence, we obtain a natural exact sequence of $G(w)$-modules

$$
0 \rightarrow X^{I_{w}} / e_{v} \rightarrow H^{1}\left(I_{w}, \operatorname{Ker} N_{v}\right) \rightarrow H^{1}\left(I_{w}, X\right) \rightarrow 0
$$


and therefore an exact sequence of abelian groups

$$
\begin{aligned}
H^{1}\left(G(w), X^{I_{w}} / e_{v}\right)^{D} \stackrel{\partial^{D}}{\longrightarrow}\left(H^{1}\left(I_{w}, X\right)^{G(w)}\right)^{D} & \rightarrow\left(H^{1}\left(I_{w}, \operatorname{Ker} N_{v}\right)^{G(w)}\right)^{D} \\
& \rightarrow\left(\left(X^{I_{w}} / e_{v}\right)^{G(w)}\right)^{D} \rightarrow 0,
\end{aligned}
$$

where $\partial: H^{1}\left(I_{w}, X\right)^{G(w)} \rightarrow H^{1}\left(G(w), X^{I_{w}} / e_{v}\right)$ is the "connecting homomorphism" appearing in the long $G(w)$-cohomology sequence arising from (14) (see, e.g., [1, end of $\S 2$, p.97, for a general description of $\partial$ ).

Lemma 4.1. There exists an isomorphism

$$
\left(H^{1}\left(I_{w}, X\right)^{G(w)}\right)^{D}=I_{w} / G_{w}^{\prime} .
$$

Proof. We will need the dual of the well-known inflation-restriction-transgression exact sequence 21, p.51, namely

$$
\begin{aligned}
H^{2}\left(G(w), X^{I_{w}}\right)^{D} \rightarrow\left(H^{1}\left(I_{w}, X\right)^{G(w)}\right)^{D} & \rightarrow H^{1}\left(G_{w}, X\right)^{D} \\
& \rightarrow H^{1}\left(G(w), X^{I_{w}}\right)^{D} \rightarrow 0 .
\end{aligned}
$$

Since $H^{1}\left(I_{w}, \mathbb{Z}\right)=0$, the exact sequence (12) induces an exact sequence of $G(w)$ modules

$$
0 \rightarrow \mathbb{Z} \rightarrow \mathbb{Z}[G(w)]^{\left[G: G_{w}\right]} \rightarrow X^{I_{w}} \rightarrow 0 .
$$

Taking $G(w)$-cohomology of the preceding exact sequence and using Shapiro's lemma together with $[5$, p.250, (as well as the periodicity of the cohomology of cyclic groups), we obtain isomorphisms

$$
H^{1}\left(G(w), X^{I_{w}}\right)^{D}=H^{2}(G(w), \mathbb{Z})^{D}=G(w)
$$

and

$$
H^{2}\left(G(w), X^{I_{w}}\right)^{D}=H^{3}(G(w), \mathbb{Z})^{D}=H^{1}(G(w), \mathbb{Z})^{D}=0 .
$$

On the other hand, taking $G_{w}$-cohomology of the exact sequence of $G_{w}$-modules

$$
0 \rightarrow \mathbb{Z} \rightarrow \mathbb{Z}\left[G_{w}\right]^{\left[G: G_{w}\right]} \rightarrow X \rightarrow 0
$$

we obtain, using Shapiro's lemma and [5], p.250, again, isomorphisms

$$
H^{1}\left(G_{w}, X\right)^{D}=H^{2}\left(G_{w}, \mathbb{Z}\right)^{D}=G_{w}^{\text {ab }} .
$$

Thus (16) is isomorphic to an exact sequence

$$
0 \rightarrow\left(H^{1}\left(I_{w}, X\right)^{G(w)}\right)^{D} \rightarrow G_{w}^{\mathrm{ab}} \rightarrow G(w) \rightarrow 0 .
$$

It is a bit tedious, although not really difficult, to check that the map $G_{w}^{\text {ab }} \rightarrow G(w)$ appearing above is the canonical projection map $G_{w} / G_{w}^{\prime} \rightarrow G_{w} / I_{w}$. This involves checking the commutativity of a certain diagram whose horizontal arrows are duals of inflation maps, and whose vertical arrows are duals of composites of connecting homomorphisms of the form $H^{1} \rightarrow H^{2}$ with integral duality isomorphisms. The lemma is now immediate.

Lemma 4.2. $\left[\left(X^{I_{w} / e_{v}}\right)^{G(w)}\right]=e_{v}^{d_{v}}\left(f_{v}, e_{v}\right)$. Further, there exists an isomorphism

$$
H^{1}\left(G(w), X^{I_{w}} / e_{v}\right)^{D}=G(w)_{e_{v}} .
$$


Proof. Since $\mathbb{Z}[G]=\mathbb{Z}\left[G_{w}\right]^{\left[G: G_{w}\right]}$ (as $G_{w}$-modules), $\mathbb{Z}\left[G_{w}\right]^{I_{w}}=\mathbb{Z}[G(w)]$ (canonically) and $\operatorname{Tor}_{1}^{\mathbb{Z}}\left(X^{I_{w}}, \mathbb{Z} / e_{v}\right)=0$, we see that (12) yields an exact sequence of $G(w)$ modules

$$
0 \rightarrow \mathbb{Z} / e_{v} \rightarrow\left(\mathbb{Z}[G(w)] \otimes \mathbb{Z} / e_{v}\right)^{\left[G: G_{w}\right]} \rightarrow X^{I_{w}} / e_{v} \rightarrow 0 .
$$

We conclude, using Shapiro's lemma, that there exists an exact sequence

$$
0 \rightarrow \mathbb{Z} / e_{v} \rightarrow\left(\mathbb{Z} / e_{v}\right)^{\left[G: G_{w}\right]} \rightarrow\left(X^{I_{w}} / e_{v}\right)^{G(w)} \rightarrow \operatorname{Hom}\left(G(w), \mathbb{Z} / e_{v}\right) \rightarrow 0 .
$$

The first assertion of the lemma is now clear. On the other hand, there exist isomorphisms

$$
\begin{aligned}
H^{1}\left(G(w), X^{I_{w}} / e_{v}\right)^{D} & =H^{2}\left(G(w), \mathbb{Z} / e_{v}\right)^{D}=\left(H^{2}(G(w), \mathbb{Z}) / e_{v}\right)^{D} \\
& =\left(H^{2}(G(w), \mathbb{Z})^{D}\right)_{e_{v}}=G(w)_{e_{v}}
\end{aligned}
$$

(to obtain the second equality, take $G(w)$-cohomology of the sequence $0 \rightarrow \mathbb{Z} \stackrel{e_{v}}{\longrightarrow}$ $\left.\mathbb{Z} \rightarrow \mathbb{Z} / e_{v} \rightarrow 0\right)$. This completes the proof.

We now return to the exact sequence (15). The map labeled $\partial^{D}$ there corresponds, under the isomorphisms of Lemmas 4.1 and 4.2, to the connecting homomorphism $c: G(w)_{e_{v}} \rightarrow I_{w} / G_{w}^{\prime}=\left(I_{w} / G_{w}^{\prime}\right) / e_{v}$ appearing in the exact sequence

$$
0 \rightarrow I_{w} / G_{w}^{\prime} \rightarrow\left(G_{w}^{\mathrm{ab}}\right)_{e_{v}} \rightarrow G(w)_{e_{v}} \stackrel{c}{\longrightarrow} I_{w} / G_{w}^{\prime}
$$

(this is the beginning of the exact sequence (2), with $m=e_{v}$, associated to the short exact sequence $\left.0 \rightarrow I_{w} / G_{w}^{\prime} \rightarrow G_{w}^{\mathrm{ab}} \rightarrow G(w) \rightarrow 0\right)$. Therefore

$$
\left[\operatorname{Coker}\left(\partial^{D}\right)\right]=\frac{\left[\left(G_{w}^{\mathrm{ab}}\right) e_{v}\right]}{\left(f_{v}, e_{v}\right)} .
$$

Now the exactness of (15) together with the first assertion of Lemma 4.2 yield the identity

$$
\left[H^{1}\left(I_{w}, \operatorname{Ker} N_{v}\right)^{G(w)}\right]=e_{v}^{d_{v}}\left[\left(G_{w}^{\mathrm{ab}}\right)_{e_{v}}\right] .
$$

Thus, by Remark 3.6, the following (simple) formula holds.

Lemma 4.3. $q\left(\delta_{v}\right)=\left[\left(G_{w_{v}}^{\mathrm{ab}}\right)_{e_{v}}\right]^{-1}$.

We now compute the global factors entering in the formula of Remark 3.10.

Since $\operatorname{Tor}_{1}^{\mathbb{Z}}(\mathbb{Z},-)=0$, there exists an exact sequence of $G$-modules

$$
0 \rightarrow X^{\vee} \otimes\left(K^{*} / \mathcal{O}_{K, S_{K}}^{*}\right) \rightarrow \mathbb{Z}[G] \otimes\left(K^{*} / \mathcal{O}_{K, S_{K}}^{*}\right) \rightarrow K^{*} / \mathcal{O}_{K, S_{K}}^{*} \rightarrow 0
$$

which is induced by the $\mathbb{Z}$-linear dual of (12). Now, taking Tate cohomology $\widehat{H}^{r}$ of the above exact sequence and using Shapiro's lemma, we obtain an isomorphism

$$
H^{1}\left(G, X^{\vee} \otimes\left(K^{*} / \mathcal{O}_{K, S_{K}}^{*}\right)\right)=\widehat{H}^{0}\left(G, K^{*} / \mathcal{O}_{K, S_{K}}^{*}\right) .
$$

Arguing similarly, we obtain an isomorphism

$$
H^{1}\left(G, X^{\vee} \otimes \mathcal{O}_{K, S_{K}}^{*}\right)=\widehat{H}^{0}\left(G, \mathcal{O}_{K, S_{K}}^{*}\right)=\mathcal{O}_{F, S}^{*} / N_{K / F}\left(\mathcal{O}_{K, S_{K}}^{*}\right) .
$$

On the other hand, (13) shows that $H^{1}(G, T(K))=F^{*} / N_{K / F}\left(K^{*}\right)$. Consequently, the map $H^{1}\left(G, X^{\vee} \otimes \mathcal{O}_{K, S_{K}}^{*}\right) \rightarrow H^{1}(G, T(K))$ corresponds to the canonical map

$$
\mathcal{O}_{F, S}^{*} / N_{K / F}\left(\mathcal{O}_{K, S_{K}}^{*}\right) \rightarrow F^{*} / N_{K / F}\left(K^{*}\right) \text {. }
$$


Thus, we have a canonical isomorphism

$$
H^{1}\left(G, X^{\vee} \otimes \mathcal{O}_{K, S_{K}}^{*}\right)^{\prime}=\left(\mathcal{O}_{F, S}^{*} \cap N_{K / F}\left(K^{*}\right)\right) / N_{K / F}\left(\mathcal{O}_{K, S_{K}}^{*}\right) .
$$

Setting

$$
W_{F, S}=\mathcal{O}_{F, S}^{*} \cap N_{K / F}\left(K^{*}\right)
$$

we conclude that

$$
\left[H^{1}\left(G, X^{\vee} \otimes \mathcal{O}_{K, S_{K}}^{*}\right)^{\prime}\right]=\left[\widehat{H}^{0}\left(G, \mathcal{O}_{K, S_{K}}^{*}\right)\right] /\left[\mathcal{O}_{F, S}^{*}: W_{F, S}\right] .
$$

Next, (12) induces an exact sequence of $G$-modules

$$
0 \rightarrow X^{\vee} \otimes \mathcal{O}_{K, S_{K}}^{*} \rightarrow \mathbb{Z}[G] \otimes \mathcal{O}_{K, S_{K}}^{*} \rightarrow \mathcal{O}_{K, S_{K}}^{*} \rightarrow 0
$$

where the right-hand map is induced by the $\mathbb{Z}$-linear map $\mathbb{Z}[G] \rightarrow \mathbb{Z}, \sigma \mapsto 1$. The preceding exact sequence induces an exact sequence

$$
0 \rightarrow\left(X^{\vee} \otimes \mathcal{O}_{K, S_{K}}^{*}\right)^{G} \rightarrow \mathcal{O}_{K, S_{K}}^{*} \stackrel{N_{\mathcal{O}}}{\longrightarrow} \mathcal{O}_{F, S}^{*},
$$

where $N_{\mathcal{O}}$ is the restriction of $N_{K / F}$ to $\mathcal{O}_{K, S_{K}}^{*}$. We conclude that

$$
\left[\left(X^{\vee} \otimes \mathcal{O}_{K, S_{K}}^{*}\right)^{G}: \mathcal{T}^{\circ}(U)\right]=\left[\operatorname{Ker}\left(N_{\mathcal{O}}\right): \mathcal{T}^{\circ}(U)\right]
$$

Remarks 4.4. (a) If (13) extends to an exact sequence

$$
0 \rightarrow \mathcal{T}^{\circ} \rightarrow R_{\mathcal{O}_{K, S_{K}} / \mathcal{O}_{F, S}}\left(\mathbb{G}_{m, \mathcal{O}_{K, S_{K}}}\right) \stackrel{N_{\mathcal{O}}}{\longrightarrow} \mathbb{G}_{m, \mathcal{O}_{F, S}}
$$

of identity components of Néron-Raynaud models (cf. [16], Lemma 3.1), then the index (20) is clearly 1.

(b) The proofs of Lemma 3.7 and Corollary 3.9, together with diagram (9) (see also Remark 3.10), show that the index

$$
\left[\left(X^{\vee} \otimes \mathcal{O}_{K, S_{K}}^{*}\right)^{G}: \mathcal{T}^{\circ}(U)\right]=\left[\widetilde{\mathcal{T}}^{\circ}(\widetilde{U})^{G}: \mathcal{T}^{\circ}(U)\right]=[\operatorname{Ker}(\gamma)]
$$

divides $\prod_{v \notin S}\left[\Phi_{v}(\kappa(v))_{\text {tors }}\right]$. But [26], Corollary 2.18, p.175, and Lemma 4.1 above show that $\left[\Phi_{v}(\kappa(v))_{\text {tors }}\right]=\left[I_{w_{v}}: G_{w_{v}}^{\prime}\right]$ for any $v \notin S$. Consequently, the index $\left[\operatorname{Ker}\left(N_{\mathcal{O}}\right): \mathcal{T}^{\circ}(U)\right]$ divides $\prod_{v \notin S}\left[I_{w_{v}}: G_{w_{v}}^{\prime}\right]$, i.e., the latter integer is an upper bound for $(20)$.

Combining Remark 3.10, Lemma 4.3 and formulas (17)-(20), we obtain Chevalley's ambiguous class number formula for a norm torus:

Theorem 4.5. Let $K / F$ be a Galois extension of global fields of degree $n$ and Galois group $G$. Let $T=R_{K / F}^{(1)}\left(\mathbb{G}_{m, K}\right)$ be the corresponding norm torus. Then

$$
\frac{\left[\left(C_{K, S_{K}}^{n-1}\right)^{G}\right]}{h_{T, F, S}}=\frac{\left[\widehat{H}^{0}\left(G, K^{*} / \mathcal{O}_{K, S_{K}}^{*}\right)\right]\left[\mathcal{O}_{F, S}^{*}: W_{F, S}\right]\left[\operatorname{Ker}\left(N_{\mathcal{O}}\right): \mathcal{T}^{\circ}(U)\right]}{\left[\widehat{H}^{0}\left(G, \mathcal{O}_{K, S_{K}}^{*}\right)\right] \prod_{v \notin S}\left[\left(G_{w_{v}}^{\mathrm{ab}}\right)_{e_{v}}\right]},
$$

where $W_{F, S}$ is the group (18). 
We now need a definition. Let $G$ be any finite group and let $M$ be a left $G$-module such that both $\widehat{H}^{0}(G, M)$ and $H^{1}(G, M)$ are finite. Set

$$
h(G, M)=\frac{\left[\widehat{H}^{0}(G, M)\right]}{\left[H^{1}(G, M)\right]} .
$$

When $G$ is cyclic, $h(G, M)$ is the well-known Herbrand quotient of $M$. We now divide Chevalley's original formula (Theorem 1.1 of the Introduction) by the formula in the preceding theorem and obtain 5

Corollary 4.6. With the notations of the theorem, we have

$$
\frac{h_{T, F, S}}{h_{F, S}}=\frac{\left[\left(C_{K, S_{K}}^{n-1}\right)^{G}: C_{K, S_{K}}^{G}\right] h\left(G, \mathcal{O}_{K, S_{K}}^{*}\right) \prod_{v \notin S}\left\{\left[\left(G_{w_{v}}^{\mathrm{ab}}\right)_{e_{v}}\right] e_{v}\right\}}{\left[\mathcal{O}_{F, S}^{*}: W_{F, S}\right] h\left(G, K^{*} / \mathcal{O}_{K, S_{K}}^{*}\right)\left[\operatorname{Ker}\left(N_{\mathcal{O}}\right): \mathcal{T}^{\circ}(U)\right],}
$$

where $h\left(G, \mathcal{O}_{K, S_{K}}^{*}\right)$ and $h\left(G, K^{*} / \mathcal{O}_{K, S_{K}}^{*}\right)$ are given by (21).

Assume now that $K / F$ is a cyclic extension of degree $n$. The exact sequence $1 \rightarrow \mathcal{O}_{K, S_{K}}^{*} \rightarrow K^{*} \rightarrow K^{*} / \mathcal{O}_{K, S_{K}}^{*} \rightarrow 1$ and Hilbert's Theorem 90 show that

$$
\begin{aligned}
h\left(G, K^{*} / \mathcal{O}_{K, S_{K}}^{*}\right) & =h\left(G, K^{*}\right) / h\left(G, \mathcal{O}_{K, S_{K}}^{*}\right) \\
& =\left[F^{*}: N_{K / F}\left(K^{*}\right)\right] / h\left(G, \mathcal{O}_{K, S_{K}}^{*}\right)
\end{aligned}
$$

(see, e.g., 1], Proposition 10, p.109). On the other hand, we have the well-known formula

$$
h\left(G, \mathcal{O}_{K, S_{K}}^{*}\right)=\frac{1}{n} \prod_{v \in S}\left[K_{w_{v}}: F_{v}\right]
$$

(see [23, proof of Theorem 8.3, p.179). Finally, $\left[\left(G_{w_{v}}^{\mathrm{ab}}\right)_{e_{v}}\right]=e_{v}$ for any $v \notin S$. Using the above, we deduce from Corollary 4.6 the following formula.

Corollary 4.7. Let $K / F$ be a cyclic extension of global fields of degree $n$ and Galois group $G$. Let $T=R_{K / F}^{(1)}\left(\mathbb{G}_{m, K}\right)$ be the corresponding norm torus. Then

$$
\frac{h_{T, F, S}}{h_{F, S}}=\frac{\left[\left(C_{K, S_{K}}^{n-1}\right)^{G}: C_{K, S_{K}}^{G}\right] \prod_{v \in S}\left[K_{w_{v}}: F_{v}\right]^{2} \prod_{v \notin S} e_{v}^{2}}{n^{2}\left[\mathcal{O}_{F, S}^{*}: W_{F, S}\right]\left[F^{*}: N_{K / F}\left(K^{*}\right)\right]\left[\operatorname{Ker}\left(N_{\mathcal{O}}\right): \mathcal{T}^{\circ}(U)\right] .}
$$

Assume now that $K / F$ is a quadratic extension, i.e., $n=2$ above. Clearly,

$$
\left[\left(C_{K, S_{K}}^{n-1}\right)^{G}: C_{K, S_{K}}^{G}\right]=1 \text {. }
$$

Let $\mu$ be the number of primes in $S$ which do not split in $K$, i.e., those $v \in S$ such that $\left[K_{w_{v}}: F_{v}\right]=2$, and let $\nu$ be the number of primes of $F$ not belonging to $S$ which ramify in $K$. Then

Thus Corollary 4.7 yields

$$
\prod_{v \in S}\left[K_{w_{v}}: F_{v}\right]^{2} \prod_{v \notin S} e_{v}^{2}=4^{\mu+\nu} .
$$

\footnotetext{
${ }^{5}$ We embed $C_{K, S_{K}}$ in $C_{K, S_{K}}^{n-1}$ diagonally.
} 
Corollary 4.8. Let $K / F$ be a quadratic extension of global fields and let $T=$ $R_{K / F}^{(1)}\left(\mathbb{G}_{m, K}\right)$ be the corresponding norm torus. Then

$$
\left[\mathcal{O}_{F, S}^{*}: W_{F, S}\right]\left[F^{*}: N_{K / F}\left(K^{*}\right)\right]\left[\operatorname{Ker}\left(N_{\mathcal{O}}\right): \mathcal{T}^{\circ}(U)\right] \cdot h_{T, F, S}=4^{\mu+\nu-1} h_{F, S},
$$

where $\mu$ is the number of primes in $S$ which do not split in $K$ and $\nu$ is the number of primes of $F$ which ramify in $K$ but are not in $S$. In particular, $h_{T, F, S}$ divides $4^{\mu+\nu-1} h_{F, S}$ if $\mu+\nu \geq 1$.

Remarks 4.9. (a) Another interesting example, which we hope to discuss at length in [9, involves the quotient torus $\mathbb{S}=R_{K / F}\left(\mathbb{G}_{m, K}\right) / \mathbb{G}_{m, F}$. This torus is dual to $R_{K / F}^{(1)}\left(\mathbb{G}_{m, K}\right)$, and is isomorphic to it if $K / F$ is cyclic (see [12], Lemma 4.1, p.201). In particular, the formula of Corollary 4.7 holds true with $\mathbb{S}$ in place of $T$ (if $K / F$ is cyclic).

(b) As noted in Remark 2.1, M.Morishita [15, Theorem, p.135, obtained a formula for the class number of the natural model $\mathcal{T}^{\prime}=R_{\mathcal{O}_{K, S_{K}} / \mathcal{O}_{F, S}}^{(1)}\left(\mathbb{G}_{m, \mathcal{O}_{K, S_{K}}}\right)$ of the norm torus $R_{K / F}^{(1)}\left(\mathbb{G}_{m, K}\right)$. Since in general the exact sequence (13) above does not extend in the sense of Remark 4.4(a) (not even in the tamely ramified case. Cf. [20, Lemma 6.7, p.28), $\mathcal{T}^{\circ}$ and $\mathcal{T}^{\prime}$ need not coincide (indeed, $\mathcal{T}^{\prime}$ need not have connected fibers). It follows that Morishita's formula and our own (see, e.g., Corollary 4.6) are not immediately comparable.

\section{Acknowledgements}

I am very grateful to D.Lorenzini for several helpful comments and for encouraging me to write Section 4. I also thank the referee for suggesting several ways to improve the original version of this work. Finally, I thank B.Kunyavskii and X.Xarles for sending me copies of [1] and [16.

\section{References}

[1] M. Atiyah and C.T.C. Wall, Cohomology of groups. In: Algebraic Number Theory (J.W.S. Cassels and A. Fröhlich, Eds.), pp.94-115. Academic Press, London, 1967.

[2] A. Borel, Some finiteness properties of adele groups over number fields. Publ. Math. IHES 16 (1963), 530.

[3] S. Bosch, and Q. Liu, Rational points of the group of components of a Néron model. Manuscripta Math. 98 (1999), 275-293.

[4] S. Bosch, Lütkebohmert, W. and Raynaud, M. Néron Models. Springer Verlag, Berlin 1989.

[5] H. Cartan and S. Eilenberg, Homological Algebra. Princeton Univ. Press, Princeton, New Jersey, 1956.

[6] C. Chevalley, La théorie des corps de classes pour les corps finis et les corps locaux. J. Fac. Sci. Tokyo 2 (1933), 365-474

[7] C. Conrad, Finiteness of class numbers for algebraic groups. Available at http://www.math.lsa.umich.edu/ bdconrad

[8] S. Edixhoven, Q. Liu, and D. Lorenzini, The p-part of the group of components of a Néron model. J. Algebraic Geom. 5 (1996), 801-813.

[9] C.D. González-Avilés, The Capitulation Problem for algebraic tori. In preparation.

[10] B. Kunyavskii, and B. Moroz, On integral models of algebraic tori and affine toric varieties. Available at http://www.cs.biu.ac.il/ kunyav/publ.html

[11] B. Kunyavskii, B. Moroz, and V. Voskresenskii, On integral models of algebraic tori. St. Petersburg Math. J. 14 (2003), 35-52. 
[12] Q. Liu and D. Lorenzini, Special fibers of Néron models and wild ramification. J. reine angew. Math. 532 (2001), 179-222.

[13] J.S. Milne, Étale cohomology. Princeton Univ. Press, Princeton, New Jersey, 1980.

[14] _ _ Arithmetic Duality Theorems. Persp. in Math. 1, Academic Press Inc., Orlando, 1986.

[15] M. Morishita, On S-class number relations of algebraic tori in Galois extensions of global fields. Nagoya Math. J. 124 (1991), 133-144.

[16] E. Nart and X. Xarles, Additive reduction of algebraic tori. Arch. Math. 57 (1991), 460-466.

[17] Ye. Nisnevich, Etale Cohomology and Arithmetic of Semisimple Groups. Thesis, Harvard University, 1982.

[18] _ , The completely decomposed topology on schemes and associated descent spectral sequences in algebraic $K$-theory. Algebraic $K$-theory: connections with geometry and topology (Lake Louise, AB, 1987). NATO Adv. Sci. Inst. Ser. C Math. Phys. Sci., 279, pp. 241-342. Kluwer Acad. Publ., Dordrecht, 1989.

[19] T. Ono, on some arithmetic properties of linear algebraic groups. Ann. of Math. 70 (1959), no. 2, 266-290.

[20] G. Pappas, and M. Rapoport, Twisted loop groups and their affine flag varieties. Preprint available at http://front.math.ucdavis.edu/0607.5130

[21] J. Shatz, Profinite groups, Arithmetic, and Geometry. Annals of Math. Studies vol. 67. Princeton Univ. Press, Princeton, 1972.

[22] G. Tamme, Introduction to Étale Cohomology. Springer-Verlag, Berlin, 1994.

[23] J. Tate, Global Class Field Theory. In: Algebraic Number Theory (J.W.S. Cassels and A. Fröhlich, Eds.), pp.162-203. Academic Press, London, 1967.

[24] V. Voskresenskii, Algebraic Groups and Their Birational Invariants. Trans. Math. Monogr. vol. 179, Amer. Math. Soc. 1998.

[25] C. Weibel, An introduction to homological algebra. Cambridge Univ. Press, 1994.

[26] X. Xarles, The scheme of connected components of the Néron model of an algebraic torus. J. reine angew. Math. 437 (1993), 167-179.

Departamento de Matemáticas, Universidad de La Serena. Av. Cisternas 1200, La Serena, Chile.

E-mail address: cgonzalez@userena.cl 